\title{
Perfil de Utilização de Imunossupressores para Profilaxia de Doença Enxerto versus Hospedeiro em Pacientes Submetidos ao Transplante de Células-Tronco Hematopoiéticas
}

doi: https://doi.org/10.32635/2176-9745.RBC.2019v65n2.148

\author{
Utilization Profile of Immunosuppressants for Graft-Versus-Host Disease Prophylaxis in Patients Submitted to Hematopoietic \\ Stem Cell Transplantation \\ Perfil de Uso de Inmunosupresores para Profilaxis de Enfermedad Injerto versus Huésped en Pacientes Sometidos al \\ Trasplante de Células Madre Hematopoyéticas
}

Luna Clara França da Silva'; Carolina Lopes Martins²; Andrea Almeida Tofani ${ }^{3}$

Resumo

Introdução: Imunossupressores apresentam alta toxicidade associada à estreita faixa terapêutica, devendo-se ter controle de níveis séricos. Assim, é necessário o estudo de utilização de medicamentos em clínicas que os utilizam, fornecendo uma visão geral de seu consumo e uso racional em uma dada população. Objetivo: Identificar o perfil de utilizaçáo de imunossupressores para profilaxia de doença enxerto versus hospedeiro em pacientes submetidos a transplante de células-tronco hematopoiéticas, em um centro de transplante de medula óssea. Método: Trata-se de um estudo transversal realizado em um centro de transplante de medula óssea brasileiro. Os imunossupressores utilizados em 2017 foram classificados segundo um sistema de classificação internacional; seu consumo, expresso em dose diária definida; e seus protocolos, analisados segundo as Diretrizes para profilaxia de doença do enxerto contra hospedeiro do Consenso da Sociedade Brasileira de Transplante de Medula Óssea de 2015. Resultados: O regime de condicionamento mieloablativo foi o mais frequente (51,7\%). O protocolo de imunossupressão mais prescrito foi ciclosporina com metotrexato (37,9\%). Dos 29 pacientes elegíveis, 23 (79,3\%) tiveram protocolos seguindo as recomendaçóes do Consenso de 2015. Metotrexato, ciclosporina intravenosa e micofenolato foram responsáveis por 85,64\% do consumo. Conclusáo: Neste trabalho, só foi possível identificar um perfil de uso de imunossupressores e realizar comparaçóes dentro da instituição, em virtude da escassez de estudos de utilizaçáo desses medicamentos. Portanto, novos estudos devem ser realizados, a fim de promover seu uso racional e elaborar políticas públicas com acesso a esses medicamentos.

Palavras-chave: Uso de Medicamentos; Transplante de Células-Tronco Hematopoéticas; Doença Enxerto-Hospedeiro; Imunossupressores; Farmacoepidemiologia.

\begin{abstract}
Introduction: Immunosuppressants have high toxicity associated to a narrow therapeutic range, and serum levels should be controlled. Thus, it is necessary to study the use of drugs in clinics that use them, providing an overview of their intake and rational use in a given population. Objective: Identify the profile of the use of immunosuppressants for prophylaxis of graft versus host disease in patients submitted to hematopoietic stem cell transplantation in a bone marrow transplant center. Method: It is a cross-sectional study performed at a Brazilian bone marrow transplant facility. The immunosuppressants used in 2017 were classified according to an international classification system, their intake expressed in defined daily dose and their protocols analyzed according to the "Consenso da Sociedade Brasileira de Transplante de Medula Óssea" of 2015. Results: The myeloablative conditioning regimen was the most frequent (51.7\%). The most prescribed immunosuppressive protocol was cyclosporine with methotrexate (37.9\%). Of the 29 eligible patients, 23 (79.3\%) had protocols following the 2015 "Consenso" recommendations. Methotrexate, intravenous cyclosporine and mycophenolate were responsible for $85.64 \%$ of the intake. Conclusion: In this study, it was only possible to identify a profile of use of immunosuppressants and compare within the institution due to the scarcity of studies about the use of these drugs. Therefore, new studies should be conducted to promote their rational use and to develop public policies with access to these drugs.

Key words: Drug Utilization; Hematopoietic Stem Cell Transplantation; Graft vs Host Disease; Immunosuppressive Agents; Pharmacoepidemiology.
\end{abstract}

Resumen

Introducción: Inmunosupresores presentan una alta toxicidad asociada a la estrecha banda terapéutica, debiendo tener control de niveles séricos y alta vigilancia en cuanto a toxicidad y efectividad. Así, es necesario el estudio de uso de medicamentos en clínicas que los utilizan, proporcionando una visión general de su consumo en una determinada población. Objetivo: Identificar el perfil de uso de Inmunosupresores para profilaxia de enfermedad injerto contra huésped en pacientes sometidos al trasplante de células madre hematopoyéticas en un centro de trasplante de médula ósea. Método: Se trata de un estudio transversal realizado en un centro brasileńo de trasplante de médula ósea. Los Inmunosupresores utilizados en 2017 se clasificaron según un sistema de clasificación internacional, su consumo expresado en Dosis Diaria Definida y sus protocolos analizados según el consenso de la sociedad brasileña de trasplante de médula ósea de 2015. Resultados: El régimen de condicionamiento mieloablativo fue el más frecuente $(51,7 \%)$. El protocolo de inmunosupresión más prescrito fue ciclosporina con metotrexato (37,9\%). De 29 pacientes elegibles, 23 (79,3\%) tuvieron protocolos siguiendo recomendaciones del consenso de 2015. Metotrexato, ciclosporina intravenosa y micofenolato fueron responsables del 85,64\% del consumo. Conclusión: En este trabajo, sólo fue posible identificar un perfil de uso de Inmunosupresores y realizar comparaciones dentro de la institución debido a la escasez de estudios de utilización de esos medicamentos. Por lo tanto, nuevos estudios deben ser realizados a fin de promover su uso racional y elaborar políticas públicas con acceso a esos medicamentos.

Palabras clave: Utilización de Medicamentos; Trasplante de Células Madre Hematopoyéticas; Enfermedad Injerto contra Huésped; Inmunosupresores; Farmacoepidemiología.

1 Serviço de Farmácia do Instituto Nacional de Câncer José Alencar Gomes da Silva (INCA). Rio de Janeiro (RJ), Brasil. Orcid iD: https://orcid.org/0000-0002-9285-2109 ${ }^{2}$ Serviço de Farmácia do INCA. Rio de Janeiro (RJ), Brasil. Orcid iD: https://orcid.org/0000-0003-3236-0732

${ }^{3}$ Serviço de Farmácia do INCA. Rio de Janeiro (RJ), Brasil. Orcid iD: https://orcid.org/0000-0003-2847-7145

Endereço para correspondência: Luna Clara França da Silva. Rua Pedro José Alves, 405 - Flamengo. Maricá (RJ), Brasil. CEP 24903795. E-mail: lunaclara.fs@gmail.com 


\section{INTRODUÇÃO}

O transplante de células-tronco hematopoiéticas (TCTH), ou transplante de medula óssea (TMO), é um tratamento realizado em pacientes com doenças hematológicas malignas e benignas, imunodeficiências, erros inatos de metabolismo, alguns tumores sólidos, além de doenças autoimunes ${ }^{1,2}$.

O TCTH apresenta taxas de morbidade e mortalidade significativas. É uma modalidade de tratamento de alto custo e envolve um tratamento farmacológico complexo ${ }^{3}$. Muitos dos protocolos de condicionamento são mieloablativos; ou seja, são regimes com doses elevadas de quimioterapia e, por isso, mielotóxicas. Em caso de pacientes com alto risco de mortalidade relacionado ao transplante, pacientes idosos, com graves comorbidades ou receptores de segundo transplante, esse tipo de condicionamento pode não ser essencial para o controle da doença ${ }^{4}$. Por conta disso, foram desenvolvidos regimes de condicionamento náo mieloablativos e de intensidade reduzida, também chamados de regime de intensidade reduzida (do inglês, reduced intensity conditioning - RIC): com graus variáveis de efeitos mielossupressores para reduzir as células malignas e com menos toxicidades hematológicas ${ }^{4,5}$.

Após o transplante, em razão da intensa imunossupressão ocasionada pelos quimioterápicos na fase de condicionamento, o paciente apresenta aplasia medular, na qual ocorre deficiência imune importante que o torna vulnerável a infecçôes bacterianas, fúngicas, virais e parasitárias, necessitando utilizar inúmeros antimicrobianos ${ }^{6}$.

Além das complicações infecciosas, os pacientes submetidos a transplante estâo sujeitos a complicaçôes não infecciosas, tais como doença veno-oclusiva hepática (DVOH), mucosite, lesão pulmonar, doença do enxerto versus hospedeiro $(\mathrm{DECH})$, entre outras. No entanto, a complicaçáo de maior gravidade e potencialmente fatal em transplante alogênico é a DECH, caracterizada como uma síndrome sistêmica que ocorre em pacientes que recebem linfócitos imunocompetentes. Nesse quadro clínico, ocorre uma reação imunológica entre o tecido do hospedeiro e os linfócitos $T$ transplantados?

Aproximadamente $50 \%$ dos pacientes que realizam transplante alogênico podem desenvolver DECH, apesar da profilaxia com imunossupressores. A mortalidade pode atingir $20 \%$ e a doença pode ter vários graus de gravidade . $^{8}$ Mesmo após longos períodos após o transplante, 60\% a $80 \%$ dos pacientes submetidos a TCTH apresentam algum grau de atividade da doença e indicação de utilizaçáo de agentes imunossupressores ${ }^{9}$. A diferença de sexo entre o doador e o receptor, idade do paciente, regime de condicionamento, protocolo de profilaxia utilizado, fonte das células progenitoras, comorbidades pré-TCTH e compatibilidade human leukocyte antigen (HLA) são alguns fatores de risco para a $\mathrm{DECH}^{9,10}$.

$\mathrm{Na}$ profilaxia da $\mathrm{DECH}$, o paciente é submetido a um protocolo clínico com imunossupressores, com a finalidade de controlar a açáo das células $\mathrm{T}$ residuais provenientes do sangue do doador evitando a rejeiçáo. $\mathrm{Na}$ década de 1980, foi estabelecida uma combinação padrão de imunossupressores para a profilaxia de $\mathrm{DECH}$, na qual utilizavam inibidor de calcineurina (tacrolimo - TCL - ou ciclosporina - CSA) em combinação com metotrexato $(\mathrm{MTX})^{11}$. Outras combinaçóes como sirolimo com TCL, CSA ou TCL com micofenolato (MMF) também são utilizadas e dependem do regime de condicionamento (mieloablativo, RIC ou não mieloablativo) e do tipo de TCTH'.

Por serem de alta toxicidade associada à estreita faixa terapêutica, esses medicamentos devem ter controle de nível sérico e alta vigilância quanto à toxicidade e à efetividade. Além disso, apresentam inúmeras interações medicamentosas, levando a um aumento ou à diminuição dos níveis séricos, podendo ocorrer toxicidade ou falha terapêutica. Portanto, devem ser frequentemente monitorados e ajustados apropriadamente, principalmente em se tratando de paciente em TCTH que utiliza esquemas terapêuticos complexos com um grande número de medicamentos ${ }^{12,13}$.

Dessa forma, faz-se necessário, em um ambiente hospitalar, o estudo de utilização de medicamentos (EUM) em clínicas que usam imunossupressores de forma profilática e terapêutica. Os EUM fornecem uma visão geral do uso de medicamentos em uma dada população, além de esclarecer métodos, objetivos e finalidades ${ }^{14}$. São úteis para a assistência farmacêutica e regulação sanitária, pois permitem identificar os grupos populacionais vulneráveis ao uso irracional e as classes terapêuticas empregadas de modo inadequado ${ }^{15,16}$. Sáo utilizados para comparar medicamentos e tratamentos, trazendo um menor custo para a instituiçáo, além de um melhor retorno ao paciente ${ }^{17}$.

Diante do exposto, o presente estudo tem como objetivo identificar o perfil de utilizaçáo de medicamentos imunossupressores para profilaxia de $\mathrm{DECH}$ em pacientes submetidos ao TCTH em um centro de referência brasileiro.

\section{MÉTODO}

Trata-se de um estudo transversal realizado em um centro de transplante de medula óssea brasileiro. Para sua execuçáo, foram utilizados dados de sistemas eletrônicos 
da instituição que contêm as prescriçóes médicas e os prontuários de pacientes internados para realização de TCTH alogênicos (aparentado, náo aparentado e haploidênticos), de 1 de janeiro a 31 de dezembro de 2017. Foram excluídos pacientes com idade menor ou igual a 18 anos.

Para análise das variáveis sociodemográficas utilizadas, foram considerados: sexo, idade, religião, escolaridade, estado civil e etnia. Para análise das variáveis clínicas dos pacientes, foram usadas as doenças de base consideradas segundo a Classificação Internacional de Doenças (CID). Para análise das variáveis que envolveram a escolha da profilaxia para DECH, utilizaram-se a doença base que originou o transplante, o tipo de transplante, a fonte do transplante e o regime de condicionamento. Para avaliação da conformidade do uso de protocolos de imunossupressão, foram tomadas por base as recomendaçóes padrão atuais para profilaxia de DECH do Consenso da Sociedade Brasileira de Transplante de Medula Óssea (SBTMO) de 2015, conforme se seguem:

Transplantes alogênicos aparentados e com condicionamentos mieloablativos: associação de um inibidor de calcineurina (CSA ou TCL) iniciado no dia (D) anterior ao TCTH (D-1) mais MTX (D+1, $\mathrm{D}+3, \mathrm{D}+6$ e $\mathrm{D}+11)$.

Transplantes alogênicos aparentados e com condicionamentos RIC ou náo mieloablativos: $\mathrm{MMF}$ a partir do D+1 e CSA ou TCL iniciado no D- $1^{12}$.

Transplantes não aparentados mieloablativos, RIC ou não mieloablativos: o ATG demonstra redução da DECHc e melhora da qualidade de vida, portanto, podem ser incluídos nesses regimes e é administrado antes do TCTH $^{18}$.

Transplantes haploidênticos mieloablativos, RIC ou não mieloablativos: a profilaxia padrão é a mesma dos aparentados RIC e não mieloablativos (CSA/
TCL + MMF), porém, iniciados do D+5 e com adição de ciclofosfamida em altas doses após a infusão do enxerto (D3 e D4).

Os imunossupressores padronizados e utilizados no estudo foram aqueles prescritos para profilaxia de $\mathrm{DECH}$ e classificados segundo o Sistema Internacional de Classificação de Medicamentos anatomical therapeutic chemical (ATC), criado pelo Norwegian Medicinal Depot (NMD), no qual organiza os medicamentos disponíveis de forma clara e divididos em grupos, e seu consumo foi expresso em dose diária definida (DDD/100 leitos/dia). Segundo a Organização Mundial da Saúde (OMS), a DDD é a dose média de manutenção assumida por dia para um medicamento usado para sua principal indicação em adultos de $70 \mathrm{Kg}^{17,19}$. A fórmula utilizada é ${ }^{20}$ : DDD/100 leitos/dia $=$ [(quantidade de medicamentos consumidos em g)/(DDD estabelecida para o medicamento (OMS) $\mathrm{x}$ período de observação em dias $\mathrm{x}$ número de leitos disponíveis taxa de ocupação)] x 100.

Para o cálculo da DDD, os dados do consumo em gramas de imunossupressores padronizados foram retirados de prescrições eletrônicas. O total de pacientes-dia foi obtido do sistema eletrônico de registro de internaçôes. De acordo com a metodologia ATC/DDD, recomendada pelo Grupo de Estudos de Utilização de Medicamentos da OMS (WHO Drug Utilization Research Group), os registros de consumo de medicamentos podem ser utilizados como fonte de dados para esses estudos. A DDD de cada medicamento estabelecida pela OMS encontra-se listada na Tabela 1 . As informações sobre taxa de ocupação foram obtidas do registro de internação da instituição.

Foi realizada a análise das variáveis categóricas e dicotômicas por meio de estatísticas descritivas e frequências relativas e absolutas realizadas no software Statistical Package for the Social Sciences (SPSS 22).

O presente estudo foi iniciado somente após aprovação pelo Comitê de Ética em Pesquisa (CEP) sob o número CAAE 90590418.8.0000.5274.

Tabela 1. Valores de DDD estabelecidos para os imunossupressores pela OMS

\begin{tabular}{|c|c|c|c|c|}
\hline Código ATC & Imunossupressor & DDD & $\begin{array}{l}\text { Unidade de } \\
\text { medida }\end{array}$ & $\begin{array}{c}\text { Via de } \\
\text { administração }\end{array}$ \\
\hline L04AD01 & Ciclosporina & 0,25 & g & VO e IV \\
\hline L04AA4 & ATG & 0,1 & g & IV \\
\hline L04AA06 & Micofenolato mofetil & 2 & $\mathbf{g}$ & Vo e IV \\
\hline L04AD02 & Tacrolimo & 5 & $\mathrm{mg}$ & VO e IV \\
\hline L04AX03 & Metotrexato & 2,5 & $\mathrm{mg}$ & VO e IV \\
\hline L01AA01 & Ciclofosfamida & NE & - & - \\
\hline
\end{tabular}

Legendas: $\mathrm{VO}=$ via oral; IV=via intravenosa; $\mathrm{ATG}=$ imunoglobulina antitimócitos (coelho); $\mathrm{NE}=$ não existe.

Fonte: ATC/DDD Index 2019/ WHO. 


\section{RESULTADOS}

Durante o ano de 2017, foram internados 211 pacientes na instituição; destes, $43(20,4 \%)$ se internaram para realização de TCTH alogênicos e, após o critério de exclusão (14 pacientes $(32,6 \%) \leq 18$ anos), $29(13,7 \%)$ pacientes foram elegíveis para o estudo. Os demais pacientes internaram para TCTH autólogo, para doaçâo de medula óssea e por intercorrências relacionadas ao TCTH.

Na Tabela 2, está descrito o perfil sociodemográfico dos pacientes que realizaram TCTH alogênicos, e as maiores prevalências dos pacientes incluídos foram sexo masculino $18(62,1 \%) ; 12(41,4 \%)$ pacientes tinham idade entre 19 e 29 anos; 19 (65,5\%) eram brancos; sete (24,1\%) tinham com ensino fundamental incompleto; $15(51,7 \%)$ eram solteiros; e 14 (48,3\%), católicos.

Da amostra avaliada de pacientes, o diagnóstico de leucemia mieloide aguda (LMA) foi o mais prevalente e

Tabela 2. Perfil sociodemográfico dos pacientes que realizaram TCTH alogênicos, 2017

\begin{tabular}{l|c|c}
\hline \multicolumn{1}{c|}{ Variáveis } & $\mathbf{n}$ & $\%$ \\
\hline Gênero & 18 & 62,1 \\
Masculino & 11 & 37,9 \\
Feminino & & \\
Idade (anos) & 12 & 41,4 \\
$19-29$ & 10 & 34,5 \\
$30-49$ & 7 & 24,1 \\
$50-60$ & & \\
Raça & 19 & 65,5 \\
Branco & 6 & 20,7 \\
Pardo & 3 & 10,3 \\
Negro & 1 & 3,4 \\
Não informado & & \\
Escolaridade & 7 & 24,1 \\
Ensino fundamental incompleto & 6 & 20,7 \\
Ensino fundamental completo & 6 & 20,7 \\
Ensino médio completo & 4 & 13,8 \\
Ensino médio incompleto & 4 & 13,8 \\
Ensino superior completo & 1 & 3,4 \\
Ensino superior incompleto & 1 & 3,4 \\
Analfabeto & & \\
Estado civil & 15 & 51,7 \\
Solteiro & 13 & 44,8 \\
Casado & 1 & 3,4 \\
Divorciado & & \\
Religião & 14 & 48,3 \\
Católico & 13 & 44,8 \\
Evangélico & 3,4 \\
Cristão & \\
Sem religião & & 3,4 \\
\hline
\end{tabular}

teve um total de 13 pacientes $(44,8 \%)$, seguido de anemia aplástica idiopática com sete $(24,1 \%)$ casos. O tipo de TCTH mais realizado foi o alogênico aparentado com 12 pacientes (41,4\%), seguido de $11(37,9 \%)$ alogênicos não aparentados e seis $(20,7 \%)$ haploidênticos. Dos 29 pacientes, apenas um fez a coleta das células-tronco hematopoiéticas $(\mathrm{CTH})$ a partir do sangue periférico $(3,4 \%)$. A taxa de mortalidade em 100 dias de transplante foi de $13,8 \%$, com um total de quatro pacientes. Os demais foram provenientes da medula óssea $28(96,6 \%)$. O regime de condicionamento mieloablativo foi o mais frequente e foi realizado em um total de 15 pacientes $(51,7 \%)$. Todos os pacientes realizaram protocolo de profilaxia para DECH com no mínimo dois imunossupressores: o mais prescrito foi a combinação de CSA com MTX, realizado em 11 pacientes $(37,9 \%)$ (Tabela 3).

Os protocolos de imunossupressão utilizados variaram de acordo com o regime de condicionamento, tipo de

Tabela 3. Perfil clínico e terapêutico dos pacientes $(n=29)$

\begin{tabular}{lccc}
\hline \multicolumn{1}{c}{ Variáveis } & $\mathbf{n}$ & $\%$ \\
\hline Doença base & & \\
C 92.0 Leucemia mieloide aguda & 13 & 44,8 \\
D 61.3 Anemia aplástica idiopática & 7 & 24,1 \\
D 46.2 Anemia refratária com & 3 & 10,3 \\
excesso de blastos & & 10,3 \\
C 91.0 Leucemia linfoide aguda & 3 & \\
D 46.3 Anemia refratária & & 3,4 \\
com excesso de blastos com & 1 & \\
transformação & & \\
D 61.0 Anemia aplástica & 1 & 3,4 \\
constitucional & 1 & 3,4 \\
C 94.5 Mielofibrose aguda & & \\
Tipo de transplante & 12 & 41,4 \\
Alogênico aparentado & 11 & 37,9 \\
Alogênico não aparentado & 6 & 20,7 \\
Haploidêntico & & \\
Fonte do transplante & 28 & 96,6 \\
Medula óssea & 1 & 3,4 \\
Sangue periférico & & \\
Regime de condicionamento & 15 & 51,7 \\
Mieloablativo & 6 & 20,7 \\
RIC & 8 & 27,6 \\
Não mieloablativo & & \\
Protocolo de imunossupressão & 11 & 37,9 \\
CSA + MTX & 7 & 24,1 \\
CSA + MTX + ATG* & 5 & 17,2 \\
CSA + MMF + CY-pós & 5 & 17,2 \\
TCL + MMF + CY-pós & 1 & 3,4 \\
\hline TCL+ MTX + ATG* & & \\
\hline
\end{tabular}

Legendas: $\mathrm{n}=$ número de casos; RIC=regime de intensidade reduzida; $\mathrm{CSA}=$ ciclosporina; $\mathrm{MTX}=$ metotrexato; $\mathrm{ATG}=$ imunoglobulina antitimócitos; $\mathrm{MMF}=$ micofenolato; $\mathrm{CY}$-pós=ciclofosfamida pós-transplante; $\mathrm{TCL}=$ tacrolimo; *em fase de condicionamento. 
TCTH e compatibilidade do transplante. A Tabela 4 descreve os pacientes de acordo com o tipo de TCTH/regime de condicionamento e protocolo de imunossupressão. Do total de pacientes elegíveis, 23 (79,3\%) tiveram, em seus protocolos de imunossupressão, as recomendaçôes-padrão descritas no Consenso SBTMO 2015.

Todos os pacientes submetidos ao TCTH alogênico aparentado com regime de condicionamento mieloablativo e RIC (11 pacientes) utilizaram a associação de CSA com MTX como profilaxia de DECH, sendo que os únicos dois que fizeram o regime RIC apresentaram-se diferentes das recomendaçóes do Consenso da SBTMO, que sugere o protocolo com CSA/TCL + MMF. Apenas um paciente fez transplante alogênico aparentado não mieloablativo e utilizou a associação de CSA com MMF e ciclofosfamida pós-transplante (CY-pós). As recomendações-padrão do Consenso SBTMO 2015 sugerem apenas a associação de CSA/TCL + MMF.

Pacientes que realizaram TCTH náo aparentado com regime de condicionamento mieloablativos utilizaram em sua maioria ( 5 pacientes $-83,3 \%$ ) a associação de CSA com MTX e imunoglobulina antitimócitos (ATG). No entanto, um $(16,7 \%)$ paciente substituiu CSA por TCL nesse mesmo protocolo. Já com relação aos três pacientes não aparentados de condicionamento não mieloablativo, dois utilizaram TCL + MMF e CY-pós (66,7\%) e um CSA + MMF + CY-pós (33,7\%), diferente das diretrizes-padrão para o tratamento da DECH segundo Consenso SBTMO 2015, no qual é sugerido CSA/TCL + MTX + ATG. O condicionamento RIC para os alogênicos não aparentados teve como protocolo de profilaxia para $\mathrm{DECH}$ a associação de CSA + MTX + ATG em seus dois pacientes.

Três pacientes que realizaram TCTH haploidênticos não mieloablativo utilizaram CSA + MMF + CY-pós (75\%). No entanto, um $(25,0 \%)$ substituiu CSA por TCL nesse mesmo protocolo. Apenas dois haploidênticos tiveram o regime RIC e todos utilizaram TCL + MMF + CY-pós.

Dos 29 pacientes, quatro pacientes $(13,8 \%)$ tiveram, por um período curto, a substituição da CSA por TCL (inibidores de calcineurina).

Um total de oito pacientes apresentaram DECH durante o período de internação (27,6\%). As relaçóes entre a ocorrência de $\mathrm{DECH}$ e os protocolos de imunossupressão foram analisadas no programa SPSS. Quatro pacientes que utilizaram CSA + MTX apresentaram DECH, obtendo um total de 27,3\% nesse grupo. Dos sete pacientes que utilizaram CSA + MTX + ATG, três (42,9\%) apresentaram DECH. Nenhum dos cinco pacientes que utilizaram a associação de CSA + MMF + CY-pós tiveram a ocorrência de DECH e apenas um (20\%) dos cinco que utilizaram TCL + MMF + Cy-pós teve DECH. O paciente que utilizou TCL + MTX + ATG apresentou DECH, porém não é possível saber se teve alguma relação com o protocolo utilizado, pois apenas um paciente utilizou a associaçáo desses imunossupressores. $\mathrm{O}$ teste exato de Fisher mostrou uma significância de $\mathrm{P}=0,294$.

O estudo quantitativo da utilizaçáo de imunossupressores foi baseado na comparação das DDD desses medicamentos ao longo do ano de 2017. A Figura 1 mostra a variaçáo do consumo mensal, expressa em DDD/100 leitos-dia no período estudado. O MTX, CSA intravenoso (IV) e MMF foram responsáveis por $85,6 \%$ do consumo dos imunossupressores selecionados, sendo que o MTX foi o de maior DDD (45,9 DDD/100 leitos-dia) ao longo do ano. É possível observar oscilaçóes durante o ano e picos de consumo nos meses de junho e outubro para esses medicamentos, acompanhando os meses com maior número de pacientes transplantados: quatro pacientes em junho e quatro em outubro. Em outros meses, o número de TCTH oscilou entre um e três pacientes.

Tabela 4. Distribuição de pacientes de acordo com o Tipo de TCTH, regime de condicionamento e protocolo de imunossupressão

\begin{tabular}{|c|c|c|c|c|c|c|}
\hline \multirow[b]{2}{*}{ Tipo de TCTH/Regime de condicionamento } & \multicolumn{5}{|c|}{ Protocolo de imunossupressão } & \multirow[b]{2}{*}{ Total } \\
\hline & $\begin{array}{c}\text { CSA + } \\
\text { MTX }\end{array}$ & $\begin{array}{c}\text { TCL + } \\
\text { MMF + } \\
\text { CY-pós }\end{array}$ & $\begin{array}{c}\text { TCL+ } \\
\text { MTX + } \\
\text { ATG }\end{array}$ & $\begin{array}{c}\text { CSA + } \\
\text { MMF + } \\
\text { CY-pós }\end{array}$ & $\begin{array}{c}\text { CSA + } \\
\text { MTX + } \\
\text { ATG }\end{array}$ & \\
\hline Alogênico aparentado mieloablativo & 9 & - & - & - & - & 9 \\
\hline Alogênico aparentado não mieloablativo & - & - & - & 1 & - & 1 \\
\hline Alogênico aparentado RIC & 2 & - & - & - & - & 2 \\
\hline Alogênico não aparentado mieloablativo & - & - & 1 & - & 5 & 6 \\
\hline Alogênico não aparentado não mieloablativo & - & 2 & - & 1 & - & 3 \\
\hline Alogênico não aparentado RIC & - & - & - & - & 2 & 2 \\
\hline Haploidêntico não mieloablativo & - & 1 & - & 3 & - & 4 \\
\hline Haploidêntico RIC & - & 2 & - & - & - & 2 \\
\hline
\end{tabular}

Legendas: $\mathrm{TCTH}=$ transplante de células-tronco hematopoiéticas; $\mathrm{n}=$ número de casos; $\mathrm{CSA}=$ ciclosporina; $\mathrm{MTX}=$ metotrexato; $\mathrm{TCL}=$ tacrolimo; $\mathrm{MMF}=$ micofenolato; CY-pós=ciclofosfamida pós-transplante; ATG=imunoglobulina antitimócitos; RIC=condicionamento de intensidade reduzida. 


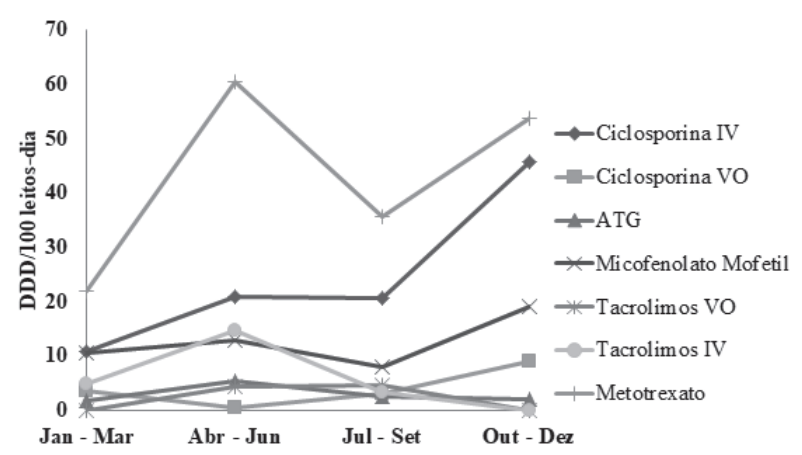

Figura 1. Variação do consumo mensal dos imunossupressores, expresso em DDD por 100 leitos-dia, 2017

Legendas: $\mathrm{IV}=$ =intravenoso; $\mathrm{VO}=$ =via oral; $\mathrm{ATG}=$ =imunoglobulina antitimócitos.

De janeiro a março, houve apenas quatro TCTH alogênicos, em que dois foram haploidênticos e que geralmente não é consenso o uso da associação padrão de CSA via intravenosa (IV) + MTX, por isso houve uma baixa da DDD desses medicamentos nesse período. Em abril, ocorreu apenas um TCTH não aparentado cujo protocolo de imunossupressão incluía TCL + MTX. Nesse período, até meados de maio, houve desabastecimento de CSA IV, tendo sido substituída por TCL IV.

Entre os meses de maio e julho, o número de TCTH alogênicos aparentados, não aparentados e haploidênticos totalizou nove e verificou-se a maior utilização da associação padrão de inibidor de calcineurina (CSA/ TCL) e MTX.

Ainda em junho, 30,4\% dos pacientes internados fizeram uso de MTX (30,4 DDD/100 leitos-dia), enquanto $13,7 \%$ dos pacientes fizeram uso de CSA IV (13,7 DDD/100 leitos-dia) e 8,4\% fizeram dose padrão de MMF (8,5 DDD/leitos-dia). Em agosto e setembro, houve apenas dois TCTH não aparentados e, de outubro a dezembro, o número de TCTH alogênicos aparentados e não aparentados aumentaram para nove, justificando assim o aumento das DDD de CSA e MTX na qual ocorreram picos no mês de outubro. Nesse mês, $27,6 \%, 20,3 \%$ e $5,6 \%$ dos pacientes fizeram uso de dose padrão de MTX, CSA IV e MMF, respectivamente. O ATG teve seu maior consumo no mês de maio $(2,3$ $\mathrm{DDD} / 100$ leitos-dia). Com relação ao TCL VO, não foi observado consumo de janeiro a abril, assim como em agosto a dezembro; tendo seu maior consumo em julho (4,6 DDD/100 leitos-dia), já o TCL IV teve aumento de consumo de janeiro a junho e uma queda em julho e não houve consumo de agosto a dezembro.

A DDD para MMF, que é usada nos TCTH aparentados com regimes náo mieloablativo e RIC e nos haploidênticos, acompanhou os aumentos de DDD nos meses de junho e outubro em função do aumento do número de TCTH e teve um aumento crescente de outubro a dezembro em razão dos TCTH haploidênticos realizados nesse período.

A DDD da CSA e TCL VO aumenta no final do ano (interrupção do medicamento parenteral passando para a formulação oral).

Náo foi possível realizar o cálculo da DDD para a ciclofosfamida, pois esta não apresenta DDD estabelecida pela ATC/DDD Index 2019/WHO.

\section{DISCUSSÃO}

Trata-se de estudo pioneiro de um centro de referência em oncologia que aborda o perfil de utilizaçáo de imunossupressores em pacientes que realizaram TCTH.

O perfil epidemiológico encontrado neste trabalho apresenta resultados semelhantes a outros estudos ${ }^{21,22}$, tais como o estudo realizado por Abreu et al. ${ }^{20}$, no qual a prevalência da população que realizou TCTH alogênico foi de pacientes do sexo masculino (59\%), católicos $(38,5 \%)$, com ensino fundamental $(33,3 \%)$, média de idade de 31,3 anos e solteiros $(53,8 \%)$.

LMA foi a doença maligna hematológica mais frequente de TCTH alogênicos, o que coincide com resultados apontados na literatura ${ }^{3,21}$. Apenas um paciente fez TCTH com coleta de sangue periférico e apresentava LMA como doença base, os demais utilizaram medula óssea. A coleta de sangue periférico tem menor incidência de recaída e a recuperação da $\mathrm{MO}$, chamada de "pega", ocorre mais rapidamente, porém, esta pode ocasionar maior frequência de DECH crônica e maior mortalidade tardia. As CTH do sangue periférico têm maiores tendências de serem usadas em doenças malignas mais avançadas ${ }^{23,24}$.

Quanto aos tipos de TCTH, há um equilíbrio entre os aparentados e não aparentados e a maioria dos condicionamentos são mieloablativos, isso justifica a prevalência do uso de CSA/TCL + MTX, que é utilizado em pacientes que realizam transplantes aparentados e não aparentados com regime mieloablativos?.

Não foi possível relacionar os protocolos de imunossupressão com a ocorrência de $\mathrm{DECH}$, pois não houve significância estatística nos resultados encontrados. Uma das possíveis hipóteses pode ser o pequeno número de participantes no estudo $(n=29)$. Em muitos centros de TCTH, utiliza-se o protocolo padrão para profilaxia de $\mathrm{DECH}$ em transplantes aparentados e não aparentados, que consiste em MTX associado com inibidores de calcineurina (CSA ou TCL) $)^{9,25,26}$. Esse tipo de profilaxia baseada nos inibidores de calcineurina (CSA e TCL) está relacionado com $25 \%$ a $40 \%$ do surgimento de $\mathrm{DECH}$ aguda e $40 \%$ a $60 \%$ de DECH crônica, o que sugere um bom controle ${ }^{18}$. Alguns estudos mostram que a 
combinação de CSA + MMF tem o intuito de reduzir a toxicidade relacionada ao uso do MTX em pacientes que realizam condicionamentos RIC e náo mieloablativo, principalmente em função de comorbidades ${ }^{25,27-29}$.

Apesar de o protocolo CSA + MTX apresentar bons resultados para TCTH alogênicos aparentados e não aparentados, nos haploidênticos ele está associado a uma incidência maior de $\mathrm{DECH}$, mesmo com a adição de ATG. Em virtude disso, houve a necessidade de se desenvolverem novos mecanismos de imunossupressão, como, por exemplo, a adição de CY-pós em doses altas no D+3 e D+4. Essa estratégia foi bem-sucedida em TCTH haploidênticos, o que levou a estudos em TCTH aparentados e náo aparentados ${ }^{18,30}$. Outros estudos sugerem a combinação de TCL + MMF + CY-pós com resultados semelhantes para TCTH haploidênticos e TCTH alogênicos aparentados e náo aparentados ${ }^{18,31-33}$.

Este é um estudo descritivo de utilização de medicamentos que teve como objetivo identificar o perfil do uso de imunossupressores para a profilaxia de $\mathrm{DECH}$, também de forma quantitativa. Para avaliar e mensurar o consumo de medicamentos em hospitais, podem ser usados vários tipos de medidas; a mais utilizada é a DDD preconizada pela OMS. Ela mostra, aproximadamente, a proporção de uma população que recebe todos os dias um tratamento farmacológico padrão e permite que os grupos de medicamentos sejam padronizados e seu uso seja comparado entre países, Regióes, locais de atenção à saúde e até mesmo dentro de uma determinada instituição ${ }^{19,34}$.

Os resultados obtidos no cálculo da DDD estão relacionados com o tipo de TCTH e o condicionamento realizado. As curvas de CSA IV, MTX e ATG foram semelhantes no estudo, essa tendência se deve ao fato de os pacientes que realizam TCTH alogênico aparentado mieloablativo poderem utilizar CSA + MTX e os não aparentados, CSA + MTX + ATG ${ }^{35}$, portanto o uso desses medicamentos pode oscilar de maneira semelhante por causa das associaçôes medicamentosas.

Cabe ressaltar que, em regimes de condicionamento mieloablativos, o paciente tem muitas chances de desenvolver mucosite oral ${ }^{36}$; em razão disso, quando o paciente é internado, são utilizados medicamentos por IV. A CSA VO, por exemplo, só é administrada aproximadamente um mês após o transplante, normalmente próxima à data da alta, pois o paciente já se recuperou da mucosite e consegue deglutir normalmente? ${ }^{9}$. Isso pode justificar o fato de a CSA VO não ter sido consumida no mês de janeiro e seu aumento em fevereiro.

A DDD para o medicamento ATG apresenta algumas pequenas variaçóes ao longo dos meses. Em alguns casos, ele foi substituído pela CY-pós em TCTH não aparentados, o que pode justificar uma baixa na DDD.
A escassez de artigos que falam de DDD de imunossupressores em TCTH foi uma justificativa para a realização do trabalho, a fim de preencher lacunas de conhecimento e contribuir para a literatura. No estudo de Gardiner et al. ${ }^{37}$, a equipe de farmácia de uma universidade australiana realizou um estudo com alguns imunossupressores utilizando a DDD, no qual mostra que, entre 2007 a 2013, a utilização de MMF, TCL e everolimus aumentou 2,7 vezes, 2,2 vezes e 2,3 vezes, respectivamente. $\mathrm{O}$ uso da CSA e sirolimus diminuiu $20 \%$ e $30 \%$, respectivamente. A partir deste estudo de DDD, notou-se que o uso de imunossupressores estấo aumentando na Austrália e no Norte da Europa e, portanto, com o aumento da sobrevida dos pacientes transplantados, esse consumo tende a aumentar ${ }^{37}$.

Uma limitação foi o pequeno número da população estudada $(n=29)$, além da delimitação de não ter sido possível calcular a DDD para a ciclofosfamida, utilizada como imunossupressão após o transplante, uma vez que não se tem referência da DDD estabelecida para esse medicamento pela ATC/DDD Index 2019/WHO.

Segundo o Guidelines for ATC classification and DDD assignment $2019^{19}$, a DDD é definida como a dose média de manutenção diária para determinado fármaco, na sua indicação principal, em adultos (a referência de peso é de $70 \mathrm{Kg}$ )". Da mesma forma, Lee e Bergman em 1989 mostraram que o uso da DDD em estudos que envolvem pacientes pediátricos causa discrepância nos resultados em função da grande diferença da magnitude da dose. A situação pode levar a uma subestimativa da exposiçáo da populaçãao ${ }^{34,38}$. Dito isso, somente pacientes adultos foram incluídos neste trabalho, já que pacientes pediátricos poderiam subestimar o cálculo da DDD. O que justifica uma redução de 32,6\% (14 pacientes pediátricos) na populaçáo do estudo.

Novas medidas como a "dias de terapia" (do inglês, days of therapy - DOT) vêm sendo adotadas para o aprimoramento das análises em algumas classes de medicamentos, como, por exemplo, os antimicrobianos. Essa ferramenta pode ser bastante útil para o seu monitoramento e análise, que, em alguns aspectos, mostra-se melhor e com maior relevância do que a $\mathrm{DDD}^{39}$. A DDD de fato é arbitrária e não leva em consideração a faixa de doses por paciente, já que ela determina uma dose em gramas, independentemente do peso. A DOT/1000 pacientes-dia náo subestima ou superestima o uso do medicamento, ela descreve o uso real por unidade de tempo e, por conta disso, muitos autores acreditam que essa medida é mais apropriada ${ }^{40}$.

Outra limitação do estudo é o fato de que as doses padrōes de imunossupressores, assim como doses de medicamentos para pediatria, são calculadas pelo 
peso do paciente. Além disso, o período de uso de imunossupressores para a profilaxia de $\mathrm{DECH}$ varia com o tipo de TCTH e pode chegar a 180 dias $^{9}$, o que sugere que a DOT poderia ser uma melhor opçáo de medida nesta análise. Neste estudo transversal, apenas se avaliou o consumo dos imunossupressores, enquanto os pacientes que realizaram TCTH alogênicos estiveram internados.

\section{CONCLUSÃO}

O presente estudo avalia o perfil epidemiológico e de utilização de imunossupressores em uma instituiçáo oncológica. Os EUM permitem avaliação das tendências de uso do medicamento, verificaçáo de seu uso racional e a comparaçấo de dados de medicamentos, interna e externamente aos locais de estudo. Neste estudo, todos os pacientes fizeram associação de no mínimo dois imunossupressores e a grande maioria seguiu as Recomendaçóes Padrão Nacional. No entanto, em relação à análise quantitativa dos imunossupressores, só foi possível identificar o perfil do seu consumo e realizar comparaçôes somente dentro da instituição. A DDD apresenta uma escassez de estudos qualitativos e quantitativos com métodos para EUM imunossupressores em TCTH e pode não ser a melhor medida de análise desses medicamentos. Com o aumento do número de pessoas transplantadas vivendo em uso de imunossupressores, pode-se esperar que essa classe de medicamentos continue a consumir parte crescente das despesas com medicamentos no futuro. Daí a importância deste estudo. Os resultados obtidos sugerem novos estudos para a escolha do melhor método de análise de consumo desses medicamentos, de maneira a garantir seu uso racional e oferecer resultados que contribuam para a elaboração de políticas públicas com acesso a esses medicamentos.

\section{CONTRIBUIÇÕES}

Luna Clara França da Silva trabalhou na concepção e planejamento do estudo, coleta de dados e redação final. Andrea Almeida Tofani e Carolina Lopes Martins trabalharam na concepção e planejamento do estudo e na revisão crítica do manuscrito. Todas as autoras aprovaram a versão final a ser publicada.

\section{DECLARAÇÃO DE CONFLITO DE INTERESSES}

Nada a declarar.

\section{FONTES DE FINANCIAMENTO}

Não há

\section{REFERÊNCIAS}

1. Pasquini R, Coutinho E. Fundamentos e biologia do transplante de células-tronco hematopoiéticas. In: Zago MA, Falcão RP, Pasquini R. Tratado de hematologia. Rio de Janeiro: Atheneu: 2013. cap. 75, p. 711-729.

2. Vigorito AC, Souza CA. Transplante de células-tronco hematopoéticas e a regeneração da hematopoese. Rev Bras Hematol Hemoter. 2009;31(4):280-284. doi: http:// dx.doi.org/10.1590/S1516-84842009005000057.

3. Gratwohl A, Baldomero H, Aljurf M, et al. Hematopoietic stem cell transplantation: a global perspective. JAMA. 2010;303(16):1617-24. doi: http://dx.doi.org/10.1001/ jama.2010.491.

4. Martino R, Caballero MD, Canals C, et al. Allogeneic peripheral blood stem cell transplantation with reducedintensity conditioning: results of a prospective multicentre study. Br J Haematol. 2001;115(3): 653-659. doi: http:// dx.doi.org/10.1046/j.1365-2141.2001.03153.x.

5. Nakamura R, Forman SJ. Reduced intensity conditioning for allogeneic hematopoietic cell transplantation: considerations for evidence-based GVHD prophylaxis. Expert Rev Hematol. 2014;7(3):407-421. doi: http:// dx.doi.org/10.1586/17474086.2014.898561.

6. Riul S, Aguillar OM. Contribuição à organização de serviços de transplante de medula óssea e a atuação do enfermeiro. Rev Latino-Am Enfermagem. 1997;5(1):49-57. doi: http://dx.doi.org/10.1590/S010411691997000100006.

7. Silva MM, Bouzas LFS, Filgueira AL. Manifestaçóes tegumentares da doença enxerto contra hospedeiro em pacientes transplantados de medula óssea. An Bras Dermatol. 2005;80(1):69-80. doi: http://dx.doi. org/10.1590/S0365-05962005000100010.

8. Molldrem JJ, Komanduri K, Wieder E. Overexpressed differentiation antigens as targets of graft-versus-leukemia reactions. Curr Opin Hematol. 2002;9(6):503-508. doi: http://dx.doi.org/10.1097/00062752-200211000-00006.

9. Moreira MCR, Vigorito AC, Tavares RCBS, et al. Diretrizes para o diagnóstico, profilaxia e tratamento de doença do enxerto contra hospedeiro aguda. Rio de Janeiro: Sociedade Brasileira de Transplante de Medula Óssea; 2015. [acesso 2018 dez. 05]. III Reunião da SBTMO de Diretrizes Brasileiras em Transplante de Células Tronco Hematopoiéticas (TCTH). Disponível em: http://www.sbtmo.org.br/aula.php?id=22.

10. Dezern AE, Luznik L, Fuchs EJ, et al. Post-transplantation cyclophosphamide for GVHD prophylaxis in severe aplastic anemia. Bone Marrow Transplant. 2011;46(7):1012-1013. doi: http://dx.doi.org/10.1038/ bmt.2010.213.

11. Cutler C, Logan B, Nakamura R, et al. Tacrolimus / sirolimus vs tacrolimus / methotrexate as GVHD prophylaxis after matched, related donor allogeneic HCT. 
Blood. 2014;124(8):1372-1377. doi: http://dx.doi. org/10.1182/blood-2014-04-567164.

12. Glotzbecker B, Duncan C, Alyea E, et al. Important Drug Interactions in Hematopoietic Stem Cell Transplantation: What Every Physician Should Know. Biol Blood Marrow Transplant. 2012;18(7):989-1006. doi: http://dx.doi. org/10.1016/j.bbmt.2011.11.029.

13. Corrêa PM, Zuckermann J, Fischer GB, et al. Immunosuppressive serum levels in allogeneic hematopoietic stem cell transplantation: pharmaceutical care contribution. Pharm Pract. 2016;14(2):683. doi: http://dx.doi.org/10.18549/PharmPract.2016.02.683.

14. Oliveira EA. Estudo da utilização de medicamentos em pacientes hipertensos e diabéticos: uma abordagem farmacoepidemiológica. Infarma. 2004;16(1-2):73-7.

15. Rozenfeld S, Valente J. Estudos de utilização de medicamentos - consideraçóes técnicas sobre coleta e análise de dados. Epidemiol Serv Saúde. 2004;13(2):115-23. doi: http://dx.doi.org/10.5123/S1679-49742004000200005.

16. Storpirtis S, Mori ALPM, Yochiy A, et al. organizadores. Farmácia clínica e atenção farmacêutica. Rio de Janeiro: Guanabara Koogan; 2013.

17. Melo DO, Ribeiro E, Storpirtis S. A importância e a história dos estudos de utilização de medicamentos. Rev Bras Cienc Farm. 2006;42(4): 475-85.

18. Moiseev IS, et al. Risk-adapted GVHD prophylaxis with post-transplantation cyclophosphamide in adults after related, unrelated, and haploidentical transplantations. Eur J Haematol. 2018;100(5):395-402. doi: http:// dx.doi.org/10.1111/ejh.13030.

19. WHO Collaborating Centre for Drug Statistics Methodology. Guidelines for ATC classification and DDD assignment 2019 [Internet]. 22nd ed. Oslo: World Health Organization; 2018 [cited 2018 Dec 26]. Available from: https://www.whocc.no/filearchive/ publications/2019_guidelines_web.pdf.

20. Onzi PS, Hoffman SP, Camargo AL. Avaliação do consumo de antimicrobianos injetáveis de um hospital privado no ano de 2009. Bras Farm. 2011;2(2):20-25.

21. Abreu MHNG, Oliveira IR, Resende RG, et al. Análise sociodemográfica e clínica de pacientes submetidos ao transplante alogênico de células-troncos hematopoiéticas. Pesqui Bras Odontopediatria Clín Integr. 2012;12(3):345-350. doi: http://dx.doi. org/10.4034/PBOCI.2012.123.07.

22. Castro Júnior CG, Gregianin LJ, Brunetto AL. Análise clínica e epidemiológica do transplante de medula óssea em um serviço de oncologia pediátrica. J Pediatria. 2003;79(5):413-22. doi: http://dx.doi.org/10.1590/ S0021-75572003000500008.

23. Pasquini R, Coutinho E. Fundamentos e biologia do transplante de células-tronco hematopoiéticas. In: Zago MA, Falcão RP, Pasquini R. Tratado de hematologia. São Paulo: Atheneu; 2013. p. 711-29.
24. Holtick U, Albrecht M, Chemnitz JM, et al. Bone marrow versus peripheral blood allogeneic haematopoietic stem cell transplantation for haematological malignancies in adults. Cochrane Database Syst Rev. 2014;20(4):CD010189. doi: http://dx.doi.org/10.1002/14651858.CD010189.pub2.

25. Ruutu T, van Biezen A, Hertenstein B, et al. Prophylaxis and treatment of GVHD after allogeneic haematopoietic SCT: a survey of centre strategies by the European Group for Blood and Marrow Transplantation. Bone Marrow Transplant. 2012;47(11):1459-1464. doi: http://dx.doi. org/10.1038/bmt.2012.45.

26. Nash RA, Antin JH, Karanes C, et al. Phase 3 study comparing methotrexate and tacrolimus with methotrexate and cyclosporine for prophylaxis of acute graft-versus-host disease after marrow transplantation from unrelated donors. Blood. 2000;96(6):2062-8.

27. Piñana JL, Valcárcel D, Fernández-Avilés F, et al. MTX or mycophenolate mofetil with CsA as GVHD prophylaxis after reduced-intensity conditioning PBSCT from HLA-identical siblings. Bone Marrow Transplant. 2010;45(9):1449-56. doi: http://dx.doi.org/10.1038/ bmt.2009.362.

28. Perkins J, Field T, Kim J, et al. A randomized phase II trial comparing tacrolimus and mycophenolate mofetil to tacrolimus and methotrexate for acute graft-versushost disease prophylaxis. Biol Blood Marrow Transplant. 2010;16(7):937-947. doi: http://dx.doi.org/10.1016/j. bbmt.2010.01.010.

29. Weber T, Niestadtkötter J, Wienke A, et al. Entericcoated mycophenolate sodium containing GvHD prophylaxis reduces GvHD rate after allogeneic HSCT. Eur J Haematol. 2016;97(3):232-238. doi: http://dx.doi. org/10.1111/ejh.12710.

30. Ringdén O. GVHD prophylaxis made safe, easy, and inexpensive. Blood. 2014;124(25): 3672-3673. doi: http://dx.doi.org/10.1182/blood-2014-10-607879.

31. Luznik L, O’Donnell PV, Symons HJ, et al. HLAhaploidentical bone marrow transplantation for hematologic malignancies using nonmyeloablative conditioning and high-dose, posttransplantation cyclophosphamide. Biol Blood Marrow Transplant. 2008; 14(6): 641-650. doi: http://dx.doi.org/10.1016/j. bbmt.2008.03.005.

32. Luznik L, Bolaños-Meade J, Zahurak M, et al. High-dose cyclophosphamide as single-agent, shortcourse prophylaxis of graft-versus-host disease. Blood. 2010;115(16):3224-3230. doi: http://dx.doi. org/10.1182/blood-2009-11-251595.

33. Luzni, L, Jalla S, Engstrom LW, et al. Durable engraftment of major histocompatibility complex-incompatible cells after nonmyeloablative conditioning with fludarabine, low-dose total body irradiation, and posttransplantation cyclophosphamide. Blood. 2001;98(12):3456-64. doi: http://dx.doi.org/10.1182/blood.v98.12.3456. 
34. Castro CGSO. Estudos de utilização de medicamentos: noçôes básicas [Internet]. Rio de Janeiro: Editora Fiocruz; 2000 [acesso 2018 out. 01]. Disponível em: http://books. scielo.org/id/zq6vb.

35. Ruutu T, Gratwohl A, de Witte T, et al. Prophylaxis and treatment of GVHD: EBMT-ELN working group recommendations for a standardized practice. Bone Marrow Transplant. 2014;49(2):168-173. doi: http:// dx.doi.org/10.1038/bmt.2013.107.

36. Cutler C, Li S, Kim HT, et al. Mucositis after allogeneic hematopoietic stem cell transplantation: a cohort study of methotrexate-and non-methotrexate-containing graftversus-host disease prophylaxis regimens. Biol Blood Marrow Transplant. 2005;11(5):383-388. doi: http:// dx.doi.org/10.1016/j.bbmt.2005.02.006

37. Gardiner KM, Tett SE, Staatz CE. Multinational Evaluation of Mycophenolic Acid, Tacrolimus, Cyclosporin, Sirolimus, and Everolimus Utilization. Ann Transplant. 2016;21:1-11. doi: http://dx.doi. org/10.12659/aot.895664.

38. Lee D, Bergman U. Studies of drug utilization. In: Strom BL, Kimmel SE, Hennessy S, editors. Pharmacoepidemiology. Nova York: Churchilli Livingstone, 1989. Part. IV; p. 379.

39. Agência Nacional de Vigilância Sanitária; Gerência de Vigilância e Monitoramento em Serviços de Saúde; Gerência Geral de Tecnologia em Serviços de Saúde. Diretriz nacional para elaboraçáo de programa de gerenciamento do uso de antimicrobianos em serviços de saúde[Internet]. Brasília, DF: ANVISA; 2017. [acesso 2018 nov. 10]. Disponível em: http://portal.anvisa.gov.br.

40. Guillot J, Lebel D, Roy H, et al. Usefulness of defined daily dose and days of therapy in pediatrics and obstetricsgynecology: a comparative analysis of antifungal drugs (2000-2001, 2005-2006, and 2010-2011). J Pediatr Pharmacol Ther. 2014;19(3):196-201. doi: http://dx.doi. org/10.5863/1551-6776-19.3.196. 